\title{
Comparison of the efficiency of two commercial kits - ELFA and Western blot in estimating the phase of Toxoplasma gondii infection in pregnant women
}

\author{
Jacek Sroka', Angelina Wójcik-Fatla', Violetta Zając', Anna Sawczyn'1, Ewa Cisak', \\ Jacek Karamon², Jacek Dutkiewicz', Iwona Bojar ${ }^{3}$ \\ ${ }^{1}$ Department of Biological Health Hazards and Parasitology, Institute of Rural Health, Lublin, Poland \\ ${ }^{2}$ Department of Parasitology, National Veterinary Research Institute, Puławy, Poland \\ ${ }^{3}$ Department for Woman Health, Institute of Rural Health, Lublin, Poland
}

Sroka J, Wójcik-Fatla A, Zając V, Sawczyn A, Cisak E, Karamon J, Dutkiewicz J, Bojar I. Comparison of the efficiency of two commercial kits - ELFA and Western blot in estimating the phase of Toxoplasma gondii infection in pregnant women. Ann Agric Environ Med. 2016; 23(4): 570-575. doi: $10.5604 / 12321966.1226848$

\begin{abstract}
Sera of 89 pregnant women were selected according to the results of ELFA IgM, IgG and avidity IgG, and tested with commercial tests IgM, IgG and avidity IgG Western Blot (WB) to compare the efficacy of both techniques in determining the phase of T. gondii infection. In total, 81 of 89 tested sera (91.0\%) were classified as positive, both in the ELFA and WB tests for the presence of anti-Toxoplasma antibodies of class IgG, indicating a past infection, while the prevalence of anti-Toxoplasma positive reactions associated with the antibodies of class IgM indicating a recent infection was much lower $-31.5 \%$ and $20.2 \%$, respectively. Sera of 81 women were also tested in the ELFA and WB tests for avidity, e.g. ability of forming high-molecular IgG antibody complexes. Low or medium results in these tests (in this study all classified as low), indicating a recent infection, were detected by ELFA and WB in $22.2 \%$ and $45.7 \%$ of the total examined samples, respectively. The Spearman's rank test for correlation, performed for recognition of quantitative data of the ELFA and WB tests (index, units or points), revealed a highly significant correlation between the ELFA and WB tests for homologous classes of antibodies, both for IgM and IgG $(p<0.00001)$. In contrast, the ELFA and WB tests for detection of anti-Toxoplasma IgM antibodies were not correlated with the ELFA and WB tests for detection of anti-Toxoplasma IgG antibodies ( $p>0.05)$, except for the WB test for IgM antibodies, which showed a significant correlation with the ELFA test for IgG antibodies $(p<0.01)$. A highly significant negative correlation between the ELFA and WB test for IgM antibodies and ELFA and WB tests for IgG avidity was demonstrated $(p<0.01)$, except for a relationship between the WB test for IgM and WB for avidity, which was not significant. Such negative correlations are theoretically expected, as strong complexes with the participation of IgG antibodies are absent in the early phase of toxoplasmosis when early antibodies of IgM class are present. Summarizing, this study indicates the high usefulness of the commercial ELFA and WB tests in serodiagnostics of toxoplasmosis in pregnant women. Special attention should be paid to parallel detection of IgM antibodies and low values in the ELFA and WB tests for IgG avidity, which indicates a recent infection which may be associated with a clinical form of congenital toxoplasmosis and damage to the foetus.
\end{abstract}

\section{Key words}

toxoplasmosis, pregnant women, seroprevalence, antibodies, IgG, IgM, ELFA, Western blot, avidity, correlation of serologic tests

\section{INTRODUCTION}

Toxoplasma gondii is an intracellular protozoan parasite of humans and warm-blooded animals. The majority of human infections result from ingestion of raw or undercooked meat containing T. gondii tissue cysts, or by ingestion of food or water contaminated with oocysts $[1,2]$. Toxoplasmosis may pose a serious public health problem, especially as a congenital infection causes cerebral and ocular damage in newborns [3]. In Poland, the frequency of congenital toxoplasmosis is estimated at 1-2 cases per 1,000 births [4]. In immunocompetent organisms, the most common is an asymptomatic form of $T$. gondii infection, manifested only by a

\footnotetext{
Address for correspondence: Dr Jacek Sroka, Department of Biological Health Hazards and Parasitology, Institute of Rural Health, Jaczewskiego 2, 20-090 Lublin, Poland.

E-mail: sroka.jacek@imw.lublin.pl

Received: 11 October 2016; accepted: 09 November 2016
}

positive serologic response. However, the latent toxoplasmosis may also exert an impact on the infected persons, including the development of behavioural changes and schizophrenia [2]. Serological examination can be of limited usefulness in cases of persons with immunosuppression. In Poland, seroprevalence in people amounts to $40-60 \%$, depending on the examined group [5].

Because of the risk of vertical Toxoplasma gondii transmission to the foetus after primary infection in pregnant women, serologic screening should be conducted repeatedly during pregnancy. As the assessment of $T$. gondii phase of infection is necessary to implement the proper treatment, the determining of $T$. gondii immunologic status, based on detection of specific antibodies of IgM, IgA, and IgG classes and avidity of IgG antibody, should be routinely applied. Detection of IgM and IgA antibodies can reveal an acute infection, whereas the presence of IgG without IgM antibody can be a sign of past infection. However, as the specific IgM 
antibodies can persist for months or even years after initial contact, the detection of IgM antibody is not sufficient for the diagnosis of an acute toxoplasmosis; much more important is determination of the avidity of Toxoplasma-specific IgG antibodies. Due to possibility of the aberration in immune response (e.g. caused by persistent IgM), determination of the phase of infection might be difficult on the basis of the classical approach and may lead to false conclusions [6]. Thus, using in the Western blot an optimized combination of recombinant antigens for the detection of IgG and IgM antibodies, as well as using different phase-specific avidity antigens, could improve the determination of the Toxoplasma infection status.

The aim of this study was to assess the usefulness of commercial kits ELFA and Western blot for determining the phase of T. gondii infection in a selected group of pregnant women.

\section{MATERIALS AND METHODS}

Collected sera. In total, 89 selected sera of pregnant women - patients of private doctor's and persons routinely tested in the Department of Health Biohazards and Parasitology, Institute of Rural Health in Lublin (2012-2014) from Lublin region (eastern Poland), were selected for the study.

All serum samples were analyzed and characterized by immunoenzymatic tests ELFA (Enzyme Linked Fluorescent Assay) for the presence of IgG and IgM antibodies against Toxoplasma gondii, and for estimation of the IgG avidity (Vidas Toxo IgG, Vidas Toxo IgM, Vidas Toxo IgG Avidity tests, bioMérieux, Marcy l'Etoile, France). To perform the tests by the ELFA technique, a Mini VIDAS device was used. Tests were used in accordance with the the manufacturer's instructions. The IgG level <4 IU (International Units)/ml was reported as negative, $>8 \mathrm{IU} / \mathrm{ml}$ was reported as positive, and ranging from $4-8 \mathrm{IU} / \mathrm{ml}$ was reported as doubtful. For IgM, the level $<0.55 \mathrm{IU} / \mathrm{ml}$ was considered negative, $>0.65$ $\mathrm{IU} / \mathrm{ml}$ was considered positive, and ranging from $0.55-0.65$ $\mathrm{IU} / \mathrm{ml}$ was considered doubtful. The avidity was determined for these serum samples in which the Vidas Toxo IgG was $\geq 8$ $\mathrm{IU} / \mathrm{ml}$. According to the manufacturer, low avidity suggests a recent infection, whereas a high avidity strongly suggests an infection of more than 4 months.

In total, 81 of 89 tested sera (91.0\%) were positive in ELFA IgG. The average reactivity of the ELFA IgG was $161.1 \mathrm{UI} / \mathrm{ml}$. Among 89 sera, 28 were positive both in IgM and IgG classes, 50 sera were positive only in IgG class and negative in IgM class, whereas 3 sera were positive in IgG class and doubtful in IgM class. Among 81 sera examined by ELFA IgG avidity, 63 sera had high IgG avidity (time post-infection $-\mathrm{tpi}>4 \mathrm{~m}$ ) and 18 sera were with low IgG avidity (tpi $<4 \mathrm{~m}$ ).

According to the results in ELFA and data from questionnaires, the tested samples included negative sera (8 samples), sera of patients suspected of having acute toxoplasmosis (IgM positive, IgG positive, and IgG low avidity; 14 samples), sera of patients with recently aquired toxoplasmosis (IgM positive, IgG positive and high IgG avidity; 16 sera), sera of patients with latent toxoplasmosis (IgM negative, IgG positive and high IgG avidity; 46 sera), and sera of patients with latent toxoplasmosis after treatment (IgM negative, IgG positive and low IgG avidity, 4 sera) (Tab. 1).
Western blot (WB) examinations. All serum samples were tested for the presence of IgG and IgM antibodies against Toxoplasma gondii and for IgG avidity by Western blot technique (commercial sets: recomLine Toxoplasma IgG, recomLine Toxoplasma IgM and recomLine Toxoplasma IgG (Avidity) (Mikrogen, Germany)). All tests were performed according to manufacturer's instructions.

Comparative analysis. Comparative analysis of results achieved in WB (reaction of individual classes of antibodies with specific antigens characteristic for phases of infection: ROP1 (66 kDa), MAG1 (65 kDa), SAG1 (30 kDa), GRA7 (29 $\mathrm{kDa}), \mathrm{GRA} 8$ (35 kDa), and in ELFA (presence of IgM and IgG antibodies and IgG avidity) was conducted.

Statistical analysis. The results were analyzed by chi-square test and Spearman test for correlation, using STATISTICA v. 5.0 package (StatSoft Inc., Tulsa, Oklahoma, USA).

\section{RESULTS}

In total, 81 of 89 tested sera were positive in WB IgG (similar to ELFA IgG results) with average reactivity - 18.5 sum of points. In total, among sera tested in $\mathrm{WB}, 18$ were positive both in IgM and IgG classes, 41 sera were positive only in IgG class and negative in IgM class, 22 sera were positive in IgG class and doubtful in IgM class, whereas 2 sera were negative in IgM class and doubtful in IgG class (Tab. 1).

Results in IgM class. Showed considerable divergences. In ELFA IgM, 28 sera were positive, 3 were doubtful and 58 were negative, whereas in WB IgM 18 were positive, 22 were doubtful and 49 were negative (Tab. 2). Much less discrepant results were found in comparison of the results of ELFA IgG and WB IgG, where only 2 sera showed discordant IgG results between WB and ELFA (doubtful/negative results, respectively) (Tab. 3).

Results in panels of sera. The comparison of ELFA and WB results (doubtful results were not taken into account) for the sera of patients with acute toxoplasmosis (14 sera) showed a discordant result for one serum in IgM and for other serum in avidity IgG. In the panel of sera from patients with recently aquired infection (16 sera), false negative results in IgM WB were found for 6 sera. The other discrepant results in ELFA and WB were also found for one serum in avidity IgG. In the panel of sera from patients with latent infection and after treatment (4 sera), the discrepant results in WB and ELFA IgM were found for one serum. Among sera from the panel of chronic stage of $T$. gondii infection (47 sera), discordant results were found in IgM ELFA and WB for 2 sera and in ELFA and WB avidity IgG for one sera (Tab. 1).

Among 81 sera examined by WB IgG avidity, 44 sera were determined as having high avidity IgG (15 sera- tpi $>12 \mathrm{~m}$ and 29 sera - tpi 6-12 m), 22 sera were with medium IgG avidity (tpi 3-6 m) and 15 sera with low IgG avidity (tpi 0-3 m) (Tab. 4).

Considering patients suspected of latent toxoplasmosis (ELFA $\geq 4 \mathrm{~m}$ and $\mathrm{WB}>6 \mathrm{~m}$ ), the proportions of high-avidity results were 63/81 (77.8\%) in ELFA and 44/81 (54.3\%) in WB (Tab. 4). 
Table 1. Comparison of the results of ELFA and Western blot (WB)

\begin{tabular}{|c|c|c|c|c|c|c|c|c|c|c|c|c|c|}
\hline & \multirow{3}{*}{$\begin{array}{l}\text { No. of } \\
\text { serum }\end{array}$} & \multicolumn{4}{|c|}{$\lg M$} & \multicolumn{4}{|c|}{$\lg G$} & \multicolumn{4}{|c|}{ Avidity } \\
\hline & & \multicolumn{2}{|c|}{ ELFA } & \multicolumn{2}{|c|}{ WB } & \multicolumn{2}{|c|}{ ELFA } & \multicolumn{2}{|c|}{ WB } & \multicolumn{2}{|c|}{ ELFA } & \multicolumn{2}{|c|}{ WB } \\
\hline & & Result* & Index & Result* & $\Sigma$ of points & Result* & $\mathrm{IU} / \mathrm{ml}$ & Result* & $\begin{array}{c}\sum \text { of } \\
\text { points }\end{array}$ & $\begin{array}{c}\text { High/ } \\
\text { Low }\end{array}$ & $\begin{array}{l}\text { Time post } \\
\text { infection } \\
\text { (months) }\end{array}$ & $\begin{array}{l}\text { High/ } \\
\text { Medium/ } \\
\text { Low }\end{array}$ & $\begin{array}{l}\text { Time post } \\
\text { infection } \\
\text { (months) }\end{array}$ \\
\hline \multirow{7}{*}{ 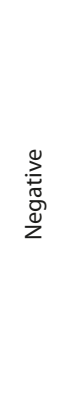 } & 1 & - & $\mathrm{N} / \mathrm{A}$ & - & 0 & - & $\mathrm{N} / \mathrm{A}$ & - & 0 & $\mathrm{~N} / \mathrm{A}$ & $\mathrm{N} / \mathrm{A}$ & N/A & $\mathrm{N} / \mathrm{A}$ \\
\hline & 2 & - & $\mathrm{N} / \mathrm{A}$ & - & 0 & - & N/A & $+/-$ & 4 & $\mathrm{~N} / \mathrm{A}$ & $\mathrm{N} / \mathrm{A}$ & $\mathrm{N} / \mathrm{A}$ & $\mathrm{N} / \mathrm{A}$ \\
\hline & 3 & - & $\mathrm{N} / \mathrm{A}$ & - & 0 & - & $\mathrm{N} / \mathrm{A}$ & - & 0 & $\mathrm{~N} / \mathrm{A}$ & N/A & $\mathrm{N} / \mathrm{A}$ & $\mathrm{N} / \mathrm{A}$ \\
\hline & 4 & - & $\mathrm{N} / \mathrm{A}$ & - & 0 & - & $\mathrm{N} / \mathrm{A}$ & - & 0 & N/A & $\mathrm{N} / \mathrm{A}$ & $\mathrm{N} / \mathrm{A}$ & $\mathrm{N} / \mathrm{A}$ \\
\hline & 5 & - & $\mathrm{N} / \mathrm{A}$ & - & 0 & - & $\mathrm{N} / \mathrm{A}$ & - & 0 & $\mathrm{~N} / \mathrm{A}$ & $\mathrm{N} / \mathrm{A}$ & $\mathrm{N} / \mathrm{A}$ & $\mathrm{N} / \mathrm{A}$ \\
\hline & 6 & - & $\mathrm{N} / \mathrm{A}$ & - & 0 & - & $\mathrm{N} / \mathrm{A}$ & $+/-$ & 4 & $\mathrm{~N} / \mathrm{A}$ & $\mathrm{N} / \mathrm{A}$ & $\mathrm{N} / \mathrm{A}$ & $\mathrm{N} / \mathrm{A}$ \\
\hline & 7 & - & $\mathrm{N} / \mathrm{A}$ & - & 0 & - & $\mathrm{N} / \mathrm{A}$ & - & 0 & $\mathrm{~N} / \mathrm{A}$ & $\mathrm{N} / \mathrm{A}$ & $\mathrm{N} / \mathrm{A}$ & $\mathrm{N} / \mathrm{A}$ \\
\hline \multirow{12}{*}{ 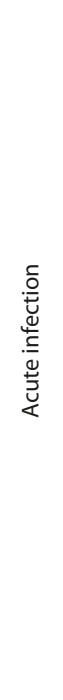 } & 9 & $+/-$ & 0.59 & + & 6 & + & 43 & + & 16 & L & $<4$ & $\mathrm{~L}$ & $0-3$ \\
\hline & 10 & + & 0.69 & $+/-$ & 6 & + & 80 & + & 21 & L & $<4$ & L & $0-3$ \\
\hline & 11 & + & 7,3 & + & 16 & + & 231 & + & 21 & $\mathrm{~L}$ & $<4$ & $\mathrm{~L}$ & $0-3$ \\
\hline & 12 & + & 0.87 & $+/-$ & 4 & + & 114 & + & 24 & L & $<4$ & M & $3-6$ \\
\hline & 13 & + & 0.86 & - & 0 & + & 71 & + & 21 & L & $<4$ & $\mathrm{~L}$ & $0-3$ \\
\hline & 14 & + & 0.65 & $+/-$ & 4 & + & 85 & + & 25 & $\mathrm{~L}$ & $<4$ & L & $0-3$ \\
\hline & 15 & + & 2.08 & + & 14 & + & 97 & + & 10 & L & $<4$ & L & $0-3$ \\
\hline & 16 & + & 2.17 & + & 14 & + & 99 & + & 10 & L & $<4$ & $\mathrm{~L}$ & $0-3$ \\
\hline & 17 & + & 1.9 & + & 6 & + & $>1200$ & + & 17 & L & $<4$ & $\mathrm{H}$ & $6-12$ \\
\hline & 18 & + & 1.3 & + & 6 & + & 52 & + & 10 & L & $<4$ & L & $0-3$ \\
\hline & 21 & + & 1.43 & + & 6 & + & 96 & + & 10 & L & $<4$ & $\mathrm{~L}$ & $0-3$ \\
\hline & 22 & + & 1.2 & + & 6 & + & 59 & + & 16 & $\mathrm{~L}$ & $<4$ & $\mathrm{~L}$ & $0-3$ \\
\hline \multirow{16}{*}{ 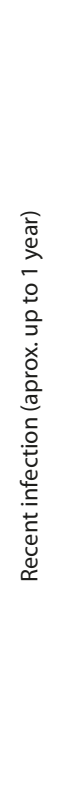 } & 23 & + & 0.86 & + & 10 & + & 167 & + & 24 & $\mathrm{H}$ & $>4$ & $\mathrm{H}$ & $6-12$ \\
\hline & 24 & + & 0.85 & $+/-$ & 4 & + & 113 & + & 25 & $\mathrm{H}$ & $>4$ & $\mathrm{H}$ & $6-12$ \\
\hline & 25 & + & 0.77 & + & 8 & + & 134 & + & 25 & $\mathrm{H}$ & $>4$ & $\mathrm{H}$ & $6-12$ \\
\hline & 26 & + & 0.75 & - & 0 & + & 73 & + & 21 & $\mathrm{H}$ & $>4$ & $\mathrm{H}$ & $6-12$ \\
\hline & 27 & + & 1.59 & - & 0 & + & 164 & + & 24 & $\mathrm{H}$ & $>4$ & $M$ & 6-12 \\
\hline & 28 & + & 2.51 & - & 0 & + & 88 & + & 19 & $\mathrm{H}$ & $>4$ & $M$ & $3-6$ \\
\hline & 29 & + & 0.95 & $+/-$ & 4 & + & $>1200$ & + & 24 & $\mathrm{H}$ & $>4$ & $\mathrm{H}$ & $6-12$ \\
\hline & 30 & $+/-$ & 0.61 & - & 0 & + & 116 & + & 21 & $\mathrm{H}$ & $>4$ & $M$ & $3-6$ \\
\hline & 31 & + & 0.8 & - & 0 & + & 74 & + & 21 & $\mathrm{H}$ & $>4$ & $\mathrm{H}$ & $6-12$ \\
\hline & 32 & + & 1.59 & - & 0 & + & 164 & + & 24 & $\mathrm{H}$ & $>4$ & $M$ & $3-6$ \\
\hline & 33 & + & 2.51 & - & 0 & + & 88 & + & 19 & $\mathrm{H}$ & $>4$ & $M$ & $3-6$ \\
\hline & 34 & + & 0.93 & $+/-$ & 4 & + & $>1200$ & + & 24 & $\mathrm{H}$ & $>4$ & $\mathrm{H}$ & $6-12$ \\
\hline & 35 & + & 0.82 & + & 10 & + & 68 & + & 21 & $\mathrm{H}$ & $>4$ & $M$ & $3-6$ \\
\hline & 36 & + & 1.02 & $+/-$ & 4 & + & 24 & + & 16 & $\mathrm{H}$ & $>4$ & $\mathrm{~L}$ & $0-3$ \\
\hline & 37 & + & 1.3 & + & 10 & + & 151 & + & 21 & $\mathrm{H}$ & $>4$ & $\mathrm{H}$ & 6-12 \\
\hline & 38 & + & 1.7 & + & 6 & + & 119 & + & 21 & $\mathrm{H}$ & $>4$ & $\mathrm{H}$ & $6-12$ \\
\hline \multirow{2}{*}{ 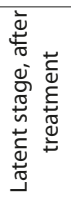 } & 39 & - & $\mathrm{N} / \mathrm{A}$ & $+/-$ & 5 & + & 128 & + & 16 & L & $<4$ & $M$ & $3-6$ \\
\hline & 40 & - & $\mathrm{N} / \mathrm{A}$ & $+/-$ & 4 & + & 122 & + & 16 & $\mathrm{~L}$ & $<4$ & $M$ & $3-6$ \\
\hline
\end{tabular}


Table 1. Comparison of the results of ELFA and Western blot (WB) (Continuation)

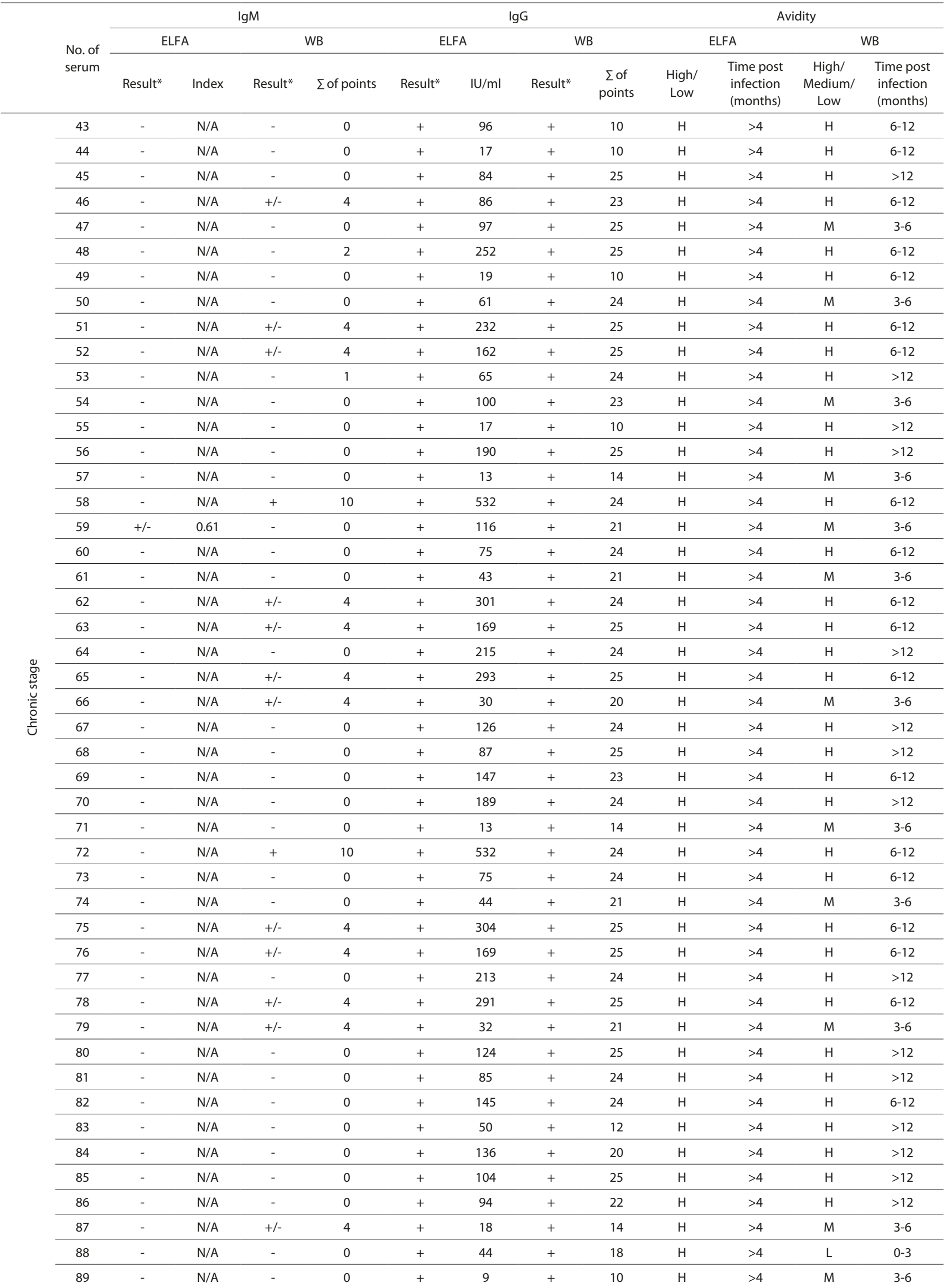


Table 2. Correlation between ELFA IgM vs. WB IgM tests with recognition of doubtful results

\begin{tabular}{llcccc}
\hline \multirow{2}{*}{ Results } & \multicolumn{4}{c}{ WB IgM } & \multirow{2}{*}{ Sum } \\
\cline { 2 - 5 } & & Positive & Negative & Doubtful & \\
\hline \multirow{3}{*}{ ELFA IgM } & Positive & 14 & 7 & 7 & 28 \\
\cline { 2 - 5 } & Negative & 3 & 40 & 15 & 58 \\
\cline { 2 - 5 } & Doubtful & 1 & 2 & 0 & 3 \\
\hline \multirow{2}{*}{ Sum } & 18 & 49 & 22 & 89 \\
\hline
\end{tabular}

Table 2a. Correlation between ELFA IgM vs. WB IgM in the positive vs. negative category*

\begin{tabular}{llccc}
\hline \multirow{2}{*}{ Results } & \multicolumn{3}{c}{ WB lgM } & Sum \\
\cline { 2 - 5 } & & Positive & Negative \\
\hline \multirow{2}{*}{ ELFA IgM } & Positive & 22 & 9 & 31 \\
\cline { 2 - 5 } & Negative & 18 & 40 & 58 \\
\hline Sum & 40 & 49 & 89 \\
\hline
\end{tabular}

Assessment of correlation: $x^{2}=11.46, p=0.0007$, correlation highly significant

*Doubtful reactions classified as positive

Tests assessed by chi-square $\left(\mathrm{x}^{2}\right)$ test with Yates correction

Table 3. Correlation between ELFA IgG versus WB IgG with recognition of doubtful results

\begin{tabular}{llcccc}
\hline \multirow{2}{*}{ Results } & \multicolumn{3}{c}{ WB IgG } & \multirow{2}{*}{ Sum } \\
\cline { 2 - 5 } & & Positive & Negative & Doubtful & \\
\hline \multirow{3}{*}{ ELFA IgG } & Positive & 81 & 0 & 0 & 81 \\
\cline { 2 - 6 } & Negative & 0 & 6 & 2 & 8 \\
\cline { 2 - 6 } & Doubtful & 0 & 0 & 0 & 0 \\
\hline \multirow{2}{*}{ Sum } & 81 & 6 & 2 & 89 \\
\hline
\end{tabular}

Table 3a. Correlation between ELFA IgG vs. WB IgG in the positive vs. negative category*

\begin{tabular}{llccc}
\hline \multirow{2}{*}{ Results } & \multicolumn{3}{c}{ WB IgG } & Sum \\
\cline { 2 - 5 } & & Positive & Negative \\
\hline \multirow{2}{*}{ ELFA IgG } & Positive & 81 & 0 & 81 \\
\cline { 2 - 5 } & Negative & 2 & 6 & 8 \\
\hline \multirow{2}{*}{ Sum } & & 83 & 6 & 89 \\
\hline
\end{tabular}

Assessment of the correlation: $\mathrm{x}^{2}=53.76, \mathrm{p}=0.0000$, correlation highly significant

*Doubtful reactions classified as positive

Tests assessed by chi-square $\left(\mathrm{X}^{2}\right)$ test with Yates correction.

Table 4. Correlation between ELFA AVI versus WB AVI tests

\begin{tabular}{lccccc}
\hline \multirow{2}{*}{ Results } & \multicolumn{3}{c}{ WB Avidity $\lg G$} & \multirow{2}{*}{ Total } \\
\cline { 3 - 5 } & & $\mathrm{H}(>6 \mathrm{~m})$ & $\mathrm{M}(3-6 \mathrm{~m})$ & $\mathrm{L}(<3 \mathrm{~m})$ & \\
\hline ELFA & $\mathrm{H}(\geq 4 \mathrm{~m})$ & 43 & 18 & 2 & 63 \\
\hline Avidity $\lg \mathrm{n}$ & $\mathrm{L}$ & $\mathrm{L}(<4 \mathrm{~m})$ & 4 & 13 & 18 \\
\hline & & 44 & 22 & 15 & 81 \\
\hline Total & & & & &
\end{tabular}

Table 4a. Correlation between ELFA AVI versus WB AVI tests when reactions MEDIUM and LOW in WB AVI were classified as LOW

\begin{tabular}{lcccc}
\hline & & \multicolumn{2}{c}{ WB AVI } & Sum \\
\cline { 2 - 5 } & & $H(>6 \mathrm{~m})$ & $\mathrm{M}+\mathrm{L}(<6 \mathrm{~m})$ & \\
\hline \multirow{2}{*}{ ELFA AVI } & $\mathrm{H}(\geq 4 \mathrm{~m})$ & 43 & 20 & 63 \\
\cline { 2 - 5 } & $\mathrm{L}(<4 \mathrm{~m})$ & 1 & 17 & 18 \\
\hline \multirow{2}{*}{ Sum } & & 44 & 37 & 81 \\
\hline
\end{tabular}

Assessment of the correlation: $X^{2}=19.73, p=0.0000$, correlation highly significant

Tests assessed by chi-square $\left(\chi^{2}\right)$ test with Yates correction
The proportion of high-avidity results obtained in ELFA AVI and WB AVI in the group of patients without specific IgM in ELFA IgM was $92 \%$ and $68 \%$, respectively. The proportion for sera with positive IgM results in ELFA and low avidity in ELFA and WB was $46.4 \%$ vs. $42.9 \%$, respectively.

In the group of patients without specific IgM in WB IgM the proportion of high-avidity in ELFA and WB was 98\% and $61 \%$, respectively. The proportion for sera with positive IgM results in WB and low avidity in ELFA and WB was $61.1 \%$ vs. $50 \%$.

A highly significant correlation $(p<0.001)$ was found between the ELFA and Western blot tests for avidity of the IgG anti-Toxoplasma antibodies when reactions MEDIUM and LOW in WB AVI were classified as LOW, both with the use of chi-square and Spearman tests (Tabs. 4a, 5). The significance level appeared to be greater when the test results were assessed by Spearman test in the units or points scale, compared to chi-square test assessed in the category 'positive versus negative' for LOW or HIGH avidity. The results indicate a high reliability of the assessed serological tests, the more so as they are produced by different companies.

Table 5 presents the results of the statistical assessment of the correlations between all the serologic tests applied in this study, performed by the Spearman's rank test with recognition of ELFA or WB potency expressed by number of units or points. The correlations between the ELFA and WB tests proved highly significant, both for IgM and IgG classes of antibody $(\mathrm{p}<0.00001)$. The ELFA test for antiToxoplasma IgM antibodies was not correlated with the ELFA and Western blot tests for detection of anti-Toxoplasma antibodies of IgG class. The Western blot test for IgM antiToxoplasma antibodies had different properties which did not show a significant correlation with the Western blot test for IgG anti-Toxoplasma antibodies, but showed a significant correlation with ELFA test for detection of anti-Toxoplasma antibodies of IgG class $(\mathrm{p}<0.01)$. This result may suggest the possibility of parallel occurrence of anti-Toxoplasma antibodies of different classes detected by different tests (Tab. 5).

Table 5. Correlation between serologic tests assessed by Spearman test

\begin{tabular}{lccc}
\hline Correlation tested & $\begin{array}{c}\text { Correlation } \\
\text { coefficient } \\
(\mathrm{r})\end{array}$ & $\begin{array}{c}\text { Probability } \\
(\mathrm{p})\end{array}$ & $\begin{array}{c}\text { Evaluation of the } \\
\text { correlation }\end{array}$ \\
\hline ELFA_IgM versus WB_IgM & 0.479 & 0.000002 & Highly significant \\
\hline ELFA_IgG versus WB_IgG & 0.678 & 0.000000 & Highly significant \\
\hline ELFA_AVI versus WB_AVI* & 0.523 & 0.000001 & Highly significant \\
\hline ELFA_IgM versus ELFA_IgG & 0.119 & 0.264 & Not significant \\
\hline ELFA_IgM versus WB_IgG & -0.120 & 0.259 & Not significant \\
\hline ELFA_IgM versus ELFA_AVI & -0.437 & 0.000044 & Highly significant \\
\hline ELFA_IgM versus WB_AVI & -0.352 & 0.00126 & Significant \\
\hline WB_IgM versus ELFA_IgG & 0.315 & 0.002593 & Significant \\
\hline WB_IgM versus WB IgG & 0.058 & 0.587 & Not significant \\
\hline WB_IgM versus ELFA_AVI & -0.492 & 0.000003 & Highly significant \\
\hline WB_IgM versus WB_AVI & -0.122 & 0.2754 & Not significant \\
\hline ELFA_IgG versus ELFA_AVI & 0.039 & 0.727 & Not significant \\
\hline ELFA_IgG versus WB_AVI & 0.421 & 0.000089 & Highly significant \\
\hline WB_IgG versus ELFA_AVI & 0.417 & 0.000104 & Highly significant \\
\hline WB_IgG versus WB-AVI & 0.506 & 0.000001 & Highly significant \\
\hline
\end{tabular}

${ }^{*} \mathrm{AVI}=\lg \mathrm{G}$ avidity 
Interesting dependencies were revealed by comparison of the ELFA and Western blot tests for detection of antiToxoplasma antibodies of IgM class with the ELFA and Western blot tests for avidity of IgG anti-Toxoplasma antibodies. It showed a highly significant negative correlation between these 2 pairs of tests ( $p<0.01$ ), except for a relationship between the Western blot test for IgM antibodies and Western blot test for avidity, which was not significant. The observed negative correlations confirm the theoretically expected dependence that strong complexes with the participation of IgG antibodies are formed in the late phase of toxoplasmosis, when early antibodies of IgM class are totally absent.

In contrast, highly significant positive correlations were found between the ELFA and Western blot tests for detection of anti-Toxoplasma antibodies of IgG class with the ELFA and Western blot tests for avidity of IgG anti-Toxoplasma antibodies $(\mathrm{p}<0.001)$, except for a relationship between the ELFA test for IgG antibodies and ELFA test for avidity, which was not significant. These correlations, also theoretically expected, could be explained by the fact that the strong IgGdependent reactions, as expressed by high numbers of units or points, may result in forming strong complexes, evidenced by the high grade of avidity.

\section{DISCUSSION}

Because congenital toxoplasmosis is usually the result of primary infection acquired during pregnancy, it is important to determine whether such an infection during pregnancy has actually occurred. The serologic examinations help to establish the immunological status and to determine the phase of T. gondii infection, which has an important meaning in therapy. Specific antibodies of IgM class are detected initially and, in most cases, IgM disappears within a few months. However, in some cases, specific IgM antibodies can still be observed during the chronic phase of infection - one year or longer. Thus, a positive IgM test result in a single serum sample can indicate not only a recently acquired infection, but also an infection acquired in the distant past, or can be considered as a false-positive result. The persistence of IgM antibodies (approximately one year and more) does not appear to have any clinical relevance, and these patients should be considered chronically infected. The interpretation of a positive IgM test result can be complicated by using several methods for its detection. Despite the wide distribution of commercial test kits to measure IgM antibodies, these tests often have low specificity, and the reported results are frequently misinterpreted [7]. Thus, it is recommended that a positive IgM test result should always be confirmed by testing in a reference laboratory.

IgG antibodies usually appear within 1-2 weeks postinfection and usually persist for life. The dye (Sabin-Feldman) test is regarded as the reference method for serodiagnosis of human toxoplasmosis; however, its application is limited by high cost and the need to use live tachyzoites, which creates the risk of laboratory infection. The most commonly used tests for the measurement of IgG antibody are: ELISA, IFA or Modified Agglutination Test (MAT). Recently, a number of tests for estimation of the avidity of Toxoplasma IgG antibodies have been introduced to help discriminate between recently acquired and distant infection. The functional affinity of IgG antibodies is low after primary contact with Toxoplasma antigen and increases during subsequent phases of infection. Protein-denaturing reagents, including urea, are used to dissociate the antibody-antigen complex. The avidity result is determined using the ratios of antibody titration curves of urea-treated and untreated samples. Diagnostic parameters, such as IgG avidity, are assessed in order to identify the time of infection $[8,9]$. The use of recombinant antigens for the avidity test could improve determining of the phase of infection [10]. The antigens ROP1, GRA7, GRA8 are recognized by specific IgG in the early phase of infection, whereas other antigens, i.e., SAG1 and MAG1, are recognize later by mature IgG [11]. The avidity test can provide a rapid identification of the latent Toxoplasma infection in pregnant women who have both IgG and IgM anti-Toxoplasma antibodies on initial testing during pregnancy. However, there are some limitations in the use of this method. Avidity assays are not conclusive in some immunocompromised patients and those treated for toxoplasmosis.

The presented study compared 2 different assays to detect T. gondii-specific IgG, IgM and IgG avidity. The results showed similar percentages of positive results for detection of IgG in ELFA and WB assays (81/89 (91\%); however, for a few sera the results in both techniques were discrepant.

IgG avidity was measured in 81 samples and was low in $46 \%$ (ELFA) and 43\% (WB) of ELFA IgM-positive samples, and in $61 \%$ (ELFA) and 50\% (WB) of WB IgM-positive samples. These results indicate a slightly more usefulness of ELFA IgM in recognizing a recent phase of Toxoplasma infection.

Comparison of the results for IgM negative and highavidity IgG sera can indicate the greater usefulness of the WB AVI test than ELFA AVI test in the recognition of latent Toxoplasma infection (mean reactivity for high avidity: $58.9 \%$ versus $87.9 \%$, respectively). The number of IgM positive sera with low IgG avidity was more frequently detected in WB than in ELFA (mean percentages: $53.5 \%$ versus 46.5 ), which may indirectly suggest the greater usefulness of WB AVI test in the recognition also of the recent phase of Toxoplasma infection. The use of WB-IgG avidity testing was therefore found to be a highly valuable diagnostic tool for determining the time of post-infection.

\section{REFERENCES}

1. Dubey J P, Jones J L. Toxoplasma gondii infection in humans and animals in the United States. Int J Parasitol. 2008; 38: 1257-1278.

2. Tenter A M, Heckeroth A R, Weiss L M. Toxoplasma gondii: from animals to humans. Int J Parasitol. 2000; 30: 1217-1258.

3. Montoya J G, Liesenfeld O. Toxoplasmosis. Lancet 2004; 363: 1965-1976.

4. Paul M, Petersen E, Szczapa J. Prevalence of congenital Toxoplasma gondii infection among newborns from the Poznań region of Poland: validation of a new combined enzyme immunoassay for Toxoplasma gondii-specific immunoglobulin A and immunoglobulin M antibodies. J Clin Microbiol. 2001; 39: 1912-1916.

5. Paul M. Potential risk factors for Toxoplasma gondii infection in cases with recently acquired toxoplasmosis. Przegl Epidemiol. 1998; 52: 447-54

6. Montoya JG. Laboratory diagnosis of Toxoplasma gondii infection and toxoplasmosis. J Infect Dis. 2002; 185 (Suppl. 1): S73-S82 doi:10.1086/338827.

7. Liesenfeld O, Press C, Montoya J G, Gill R, Isaac-Renton J L, Hedman $\mathrm{K}$, Remington J S. False-positive results in immunoglobulin M (IgM) Toxoplasma antibody tests and importance of confirmatory testing: the Platelia toxo IgM test. J Clin Microbiol. 1997; 35: 174-178.

8. Lappalainen M, Hedman K. Serodiagnosis of toxoplasmosis. The impact of measurement of IgG avidity. Ann Ist Super Sanita. 2004; 40: 81-88.

9. Liesenfeld O, Montoya J G, Kinney S, Press C, Remington J S. Effect of testing for IgG avidity in the diagnosis of Toxoplasma gondii infection in pregnant women: experience in a US reference laboratory. J Infect Dis. 2001; 183: 1248-53.

10. Holec-Gasior L. Toxoplasma gondii recombinant antigens as tools for serodiagnosis of human toxoplasmosis-the current status of studies. Clin Vaccine Immunol. 2013; 20: 1343-1351.

11. Pfrepper K, Enders G, Gohl M, Krczal D, Hlobil H, Wassenberg D, Soutschek E. Seroreactivity to and avidity for recombinant antigens in toxoplasmosis. Clin Diagn Lab Immunol. 2005; 12: 977-82. 